\title{
Intervalos de confianza para valores propios en el análisis de correspondencias a partir de una muestra probabilística ${ }^{1}$
}

\author{
Confidence Interval for eigenvalues in Analysis of correspondence from \\ a probabilistic sample \\ Javier Ramírez Montoya ${ }^{a}$ \\ javier.ramirez@sunisucre.edu.co \\ Guillermo Martínez Flórez ${ }^{\mathrm{b}}$ \\ gmartinez@correo.unicordoba.edu.co \\ Stalyn Guerrero Gómez ${ }^{\mathrm{c}}$ \\ stalynguerrero@usantotomas.edu.co
}

\section{Resumen}

En este artículo se comparan los intervalos de confianza para los valores propios en el análisis de correspondencia a partir de una muestra probabilística propuesto en (Ramirez 2010), para lo cual se utiliza un diseño de muestreo probabilístico $p($. y estrategias de remuestreo como es el caso de Bootstrap-t y Jackknife-delete I. Además se utilizan los intervalos de asintóticos de Anderson, ya que son utilizados frecuentemente en la práctica. Esta comparación se basa en la longitud y tasas de cobertura de los intervalos de confianza.

Palabras clave: Análisis de correspondencias, Bootstrap, intervalos de confianza, Jacknife.

\begin{abstract}
This work compares the confidence intervals for the eingenvalues in the analysis of correspondence from a probabilistic sample proposed in (Ramirez 2010) using a probabilistic sampling design $p($.$) , and strategies of resampling as the case of$ Bootstrap and Jackknife-delete I, also used Anderson Asymptotics intervals, as they are used frequently in practice, this comparison is based on the length and coverage of the confidence intervals.
\end{abstract}

Keywords: Analysis of correspondences,Bootstrap, confidence intervals, Jacknife.

\section{Introducción}

Un requisito fundamental en el análisis de correspondencia a partir de una muestra probabilística es la obtención de valores propios que permita, entre otras cosas, la obtención de las estimaciones de las coondgnadas factoriales de los puntos filas y de los puntos columnas sobre los ejes factoriales. Con ello se obtiene la interpretación de las asociaciones entre las variables categóricas. Con relación a la estimación de los valores propios en un análisis de correspondencia bajo una muestra probabilística de diseño $p($.$) , el problema de la aplicación del análisis de correspondencia a$ partir de una muestra probabilística radica en la verificación de la calidad de las estimaciones de los valores propios.

En muchas ocasiones, resulta imposible expresar de manera explícita el estimador de algunos parámetros que intervienen en el modelo estadístico, debido al desconocimiento de la distribución de estos. Por tal razón, las medidas de la calidad 
con los intervalos de confianza propuestos bajo esta metodología; en la sección 4 se describen los diseños de muestreo probabilístico MAS, $\pi \mathrm{PT}$ y $\pi \mathrm{PT}-\mathrm{MAS}$, en la sección 5 se hace una descripción de los escenarios de simulación y resultados; finalmente se presentan las conclusiones en la sección 6 .

\section{Análisis de correspondencias a partir de una mues- tra probabilística}

(Ramirez 2010) propone una metodología para realizar el procedimiento de estimación de los elementos de base en el análisis de correspondencias, enfocado fundamentalmente en las estimación de los valores propios, mediante el $\pi$ estimador de Horvitz-Thompson. Esto se hace con base en el muestreo de poblaciones finitas y el principio de expansión para estimar el total, reflejado en incrementar la importancia de los elementos de la muestra.

\subsection{AC Simple}

Dada la población $U=\{1, \ldots, N\}$. Suponga que a los elementos de $U$ se le miden 2 variables, digamos $Z_{1}$ y $Z_{2}$, con $p_{1}$ y $p_{2}$ modalidades respectivamente. La matriz de datos, resultado de la medición de las variables sobre los $N$ individuos es como sigue:

$$
Z=\left[Z_{1} \vdots Z_{2}\right]
$$

La matriz por diagonalizar es:

$$
\widehat{S}=\widehat{F}^{\prime} \widehat{D}_{p 1}^{-1} \widehat{F} \widehat{D}_{p 2}^{-1}
$$

Con $\widehat{F}$ la matriz de frecuencias relativas estimada, $\widehat{D}_{p 1}$ la matriz diagonal de perfiles fila y $\widehat{D}_{p 2}$ la matriz diagonal de perfiles columna, para el caso simple, los valores propios estimados se encuentran resolviendo el polinomio característico:

$$
\left|\widehat{S}-\widehat{\lambda} I_{p 2}\right|=(-1)^{p 2}\left(\widehat{\lambda}^{p 2}+\widehat{b}_{p-1} \widehat{\lambda}^{p 2-1}+\cdots+\widehat{b}_{1} \widehat{\lambda}+\widehat{b}_{0}\right)=0
$$

Donde

$$
\widehat{b}_{r}=f\left(\widehat{k}_{11}, \widehat{k}_{12}, \ldots, \widehat{k}_{1 p 2}, \widehat{k}_{21}, \widehat{k}_{22}, \ldots, \widehat{k}_{2 p 2}, \ldots, \widehat{k}_{p_{1} 1}, \widehat{k}_{p_{1} 2}, \ldots, \widehat{k}_{p_{1} p_{2}}\right)
$$

resultan ser funciones de totales o dominios estimados, los cuales se proponen mediante: 


$$
\widehat{k}_{i j \pi}=\sum_{l \in s} \frac{z_{i j l}}{\pi_{l}}
$$

Donde, $z_{i j l}$ corresponde a una variable dicótoma, si el individuo $l$ selecciona la modalidad $i$ de la pregunta $p_{1}$ y $j$ de la pregunta $p_{2}$ corresponde el valor 1 y 0 cuando no sucede, bajo un diseño de muestreo probabilístico $p($.$) con probabilidades de$ inclusión $\pi_{l}$.

\subsection{AC múltiple}

Para el caso múltiple, a través de un $\pi$ estimador basado en una muestra probabilística $s$ de tamaño $n$ corresponden a $m$ variables con $p_{1}, \ldots, p_{m}$ modalidades respectivamente, la matriz por diagonalizar es:

$$
\begin{aligned}
S=F^{\prime} D_{N}^{-1} F D_{p}^{-1} & =\frac{1}{m} Z^{\prime} Z D^{-1}=\frac{1}{m} B D^{-1} \\
\widehat{t}_{z j^{\prime}} & =\sum_{i \in s} \frac{z_{i j^{\prime}}}{\pi_{i}}
\end{aligned}
$$

y

$$
\widehat{t}_{z j j^{\prime}}=\sum_{i \in s} \frac{z_{i j} z_{i j^{\prime}}}{\pi_{i}}
$$

se puede establecer que un estimador aproximado para $\lambda$ es de la forma

$$
\widehat{\lambda}=f\left(\widehat{t}_{z_{1}}, \ldots, \widehat{t}_{z_{j^{\prime}}}, \ldots, \widehat{t}_{z_{p}}, \ldots, \widehat{t}_{z_{11}}, \ldots, \widehat{t}_{z_{j j^{\prime}}}, \ldots, \widehat{t}_{z_{p p}}\right)
$$

resultado de resolver el polinomio característico estimado a partir de la muestra $s$ de la matriz

$$
\widehat{S}=Z_{n}^{\prime} \Pi^{-1} Z_{n} \widehat{D}^{-1}=\widehat{B} \widehat{D}^{-1}
$$

dado por

$$
p(\lambda)=|\widehat{S}-\widehat{\lambda} I|=(-1)^{p}\left(\widehat{\lambda}^{p}+\widehat{b}_{p-1} \widehat{\lambda}^{p-1}+\cdots \widehat{b}_{1} \widehat{\lambda}+\widehat{b}_{0}\right)
$$

En efecto, la construcción de elementos de base que componen el análisis de correspondencias a partir de una muestra probabilística, requieren una buena estimación de los valores propios y sus vectores asociados.

\section{Intervalos de confianza}

\subsection{Intervalos de confianza Anderson a partir de una muestra probabilística}

Sean $\lambda_{1}>\lambda_{2}>\ldots>\lambda_{p}$ los valores propios de la matriz por diagonalizar $S$ en el análisis de correspondencia con vectores propios asociados $\beta_{1}, \beta_{2}, \ldots, \beta_{p}$ or- 
tonormales. Sean $\hat{\lambda}_{1}, \hat{\lambda}_{2}, \ldots, \hat{\lambda}_{p}$ los valores propios en obtenidos en el análisis de correspondencia a partir de una muestra probabilística.

Se puede, entonces, mostrar que:

$$
\hat{\lambda}_{i} \underset{n \rightarrow \infty}{\sim} N\left(\lambda_{i}, \frac{2 \lambda_{i}^{2}}{n}\right)
$$

Donde $\hat{\lambda}_{i}$ es un estimador consistente de $\lambda_{i}$. Así, mediante el teorema de Chebyshev, se tiene que:

$$
\lim _{n \longrightarrow \infty} P\left(\left|\hat{\lambda}_{i}-\lambda_{i}\right|<\varepsilon\right)=1, \forall \varepsilon>0
$$

Teniendo en cuenta (9) obtenemos que:

$$
\begin{gathered}
P\left[-Z_{\alpha / 2}<\sqrt{n} \frac{\left(\hat{\lambda}_{i}-\lambda_{i}\right)}{\sqrt{2} \lambda_{i}}<Z_{\alpha / 2}\right]=1-\alpha \\
P\left[-\sqrt{2} Z_{\alpha / 2} \lambda_{i}<\sqrt{n}\left(\hat{\lambda}_{i}-\lambda_{i}\right)<\sqrt{2} Z_{\alpha / 2} \lambda_{i}\right]=1-\alpha
\end{gathered}
$$

Entonces resolviendo tenemos:

$$
P\left[\frac{\hat{\lambda}_{i}}{1+\sqrt{\frac{2}{n}} Z_{\alpha / 2}}<\lambda_{i}<\frac{\hat{\lambda}_{i}}{1-\sqrt{\frac{2}{n}} Z_{\alpha / 2}}\right]=1-\alpha
$$

Por lo tanto, un intervalo de confianza de $100(1-\alpha) \%$ para los valores propios $\lambda_{i}$ en el análisis de correspondencia es:

$$
\left[\frac{\hat{\lambda}_{i}}{1+\sqrt{\frac{2}{n}} Z_{\alpha / 2}}, \frac{\hat{\lambda}_{i}}{1-\sqrt{\frac{2}{n}} Z_{\alpha / 2}}\right]
$$

\subsection{Intervalos de confianza Bootstrap a partir de una mues- tra probabilística}

Teniendo en cuenta la importancia de utilizar el método de remuestreo Bootstrap para estimar valores propios, (Milan 1995), realiza una aplicación del Bootstrap paramétrico a modelos que incorporan valores singulares, donde se evidencian discusiones importantes sobre el efecto de la variación de muestreo en las estimaciones.

Para estimar los valores propios mediante el método el remuestreo Bootstrap paramétrico, se realizan los siguientes pasos:

1. Dada la muestra de tamaño $n$, calcular $\hat{\lambda}$. La distribución de esta muestra se considera equivalente a la distribución de la población y $\hat{\lambda}$ es el estimador muestral del parámetro poblacional $\lambda$. 
2. Generar $B$ muestras Bootstrap de tamaño $n$ mediante muestreo con reemplazamiento de la muestra original y calcular los correspondientes valores $\hat{\lambda}^{* 1}, \hat{\lambda}^{* 2}, \ldots, \hat{\lambda}^{* B}$ para cada una de las $B$ muestras Bootstrap.

3. Estimar el error estándar del parámetro estimado $\hat{\lambda}$ calculando la desviación estándar de las $B$ réplicas Bootstrap.

Así, obtenemos que el error estándar es este

$$
\sigma_{\lambda}^{*}=\sqrt{\frac{\sum_{b=1}^{B}\left(\lambda^{b^{*}}-\overline{\hat{\lambda}}^{*}\right)^{2}}{(B-1)}}=\sigma_{\text {BOOT }}
$$

Donde

$$
\overline{\hat{\lambda}}^{*}=\frac{1}{B} \sum_{b=1}^{B} \lambda^{b^{*}}
$$

corresponde al promedio de los valores propios calculados en cada remuestra. Entonces un intervalo de confianza para los valores propios en el análisis de correspondencia está dado por:

$$
\left[\hat{\lambda}^{*}-Z_{\alpha / 2} \sigma_{\lambda}^{*}, \hat{\lambda}^{*}+Z_{\alpha / 2} \sigma_{\lambda}^{*}\right]
$$

Donde $\sigma_{\lambda}^{*}$ corresponde al error estandar Bootstrap dado en (14)

\subsection{Intervalos de confianza Jacknife a partir de una muestra probabilística}

Dada la cantidad de parámetros por calcular para estimar la varianza de HorvitzThompson se estudian los métodos de Jackknife y Bootstrap para la estimación de la varianza, los cuales son usados para este tipo de situaciones dada su simplicidad de cálculo y a los supuestos para su aplicación; por lo tanto, la estimación para la varianza de $\widehat{\lambda}$, según el método Jackknife presentado en (Wolter 1985) para parámetro de interés, en efecto para el análisis de correspondencias a partir de una muestra probabilística se propone como:

$$
v_{j k}=\frac{n-1}{n} \sum_{l=1}^{n}\left(\widehat{\lambda}_{n-1, l}-\frac{1}{n} \sum_{l^{\prime}=1}^{n} \widehat{\lambda}_{n-1, l^{\prime}}\right)^{2}
$$

Luego este estimador se conoce como el estimador Jackknife (delete-1) de $V\left(\widehat{\lambda}_{n}\right)$, donde:

$$
\widehat{\lambda}_{n-1, l}=f\left(\widehat{k}_{11 \pi_{(n-1)}}, \widehat{k}_{12 \pi_{(n-1)}}, \ldots, \widehat{k}_{p_{1} 1 \pi_{(n-1)}}, \widehat{k}_{p_{1} 2 \pi_{(n-1)}}, \ldots, \widehat{k}_{p_{1} p_{2} \pi_{(n-1)}}\right)
$$


y

$$
\widehat{k}_{i j \pi(n-1, l)}=\sum_{l^{\prime} \in S-\{l\}} \frac{z_{i j l^{\prime}}}{\pi_{l^{\prime}}}
$$

Es decir, $\widehat{\lambda}_{n-1, l}$ es el estimador del valor propio correspondiente, basado en la muestra de tamaño $n-1$ que resulta luego de eliminar el individuo $l$-ésimo de la muestra y $\widehat{k}_{i j \pi(n-1, l)}$ es la estimación de un dominio eliminado la misma observación.

Para determinar la consistencia del estimador de la varianza mediante el método Jackknife en el análisis de correspondencias a partir de una muestra probabilística. Suponemos una muestra aleatoria iid con distribución $p$-dimensional $F$. Además, sea $\lambda=\lambda_{n}\left(X_{1}, \ldots, X_{n}\right)$ la estadística de interés, es decir, el valor propio, bajo condiciones de regularidad y sea $\Sigma=\operatorname{var}(\lambda)$ conocida:

$$
\left(\lambda_{n}-\lambda\right) / \sigma_{n} \stackrel{d}{\rightarrow} N(0,1)
$$

Donde $N(0,1)$ denota la distribución de una variable aleatoria normal estandar, para $\lambda=g(\lambda)$ :

$$
\sigma_{n}^{2}=n^{-1} \nabla g(\lambda)^{\prime} \Sigma \nabla g(\lambda)
$$

y $\nabla g$ el gradiente de g. $\sigma_{n}^{2}$ es la varianza de $\lambda_{n}$, así:

$$
\left(\lambda_{n}-\lambda\right) / \sqrt{v_{j k}} \rightarrow_{d} N(0,1)
$$

Este es un resultado útil para inferencias estadísticas de muestras grandes. Entonces un intervalo de confianza para los valores propios mediante la técnica Jackknife está dado por:

$$
\left[\widehat{\lambda}_{n-1, l}-Z_{\alpha / 2} \sqrt{v_{j k}}, \widehat{\lambda}_{n-1, l}+Z_{\alpha / 2} \sqrt{v_{j k}}\right]
$$

Donde $v_{j k}$ esta dado en (17)

\subsection{Estimación mediante muestreo aleatorio simple (MAS)}

Bajo un diseño de muestreo probabilístico (MAS), con tamaño de muestra fijo en el que $\pi_{k}=\frac{n}{N}$ para todo $k=1,2, \ldots, N$ y $\pi_{k l}=\frac{n(n-1)}{N(N-1)}$ para $k \neq l, k, l=1,2, \ldots, N$ (Sarndal 2003). Luego, en el análisis de correspondencias a partir de una muestra probabilística del espacio $U=\{1, \ldots, N\}$, en el caso de $m$ variables con $p_{1}, \ldots, p_{m}$ modalidades, respectivamente, se puede establecer un estimador aproximado para el valor propio $\lambda$ como:

$$
\widehat{\lambda}=f\left(\widehat{t}_{z_{1}}, \ldots, \widehat{t}_{z_{j^{\prime}}}, \ldots, \widehat{t}_{z_{p}}, \ldots, \widehat{t}_{z_{11}}, \ldots, \widehat{t}_{z_{j j^{\prime}}}, \ldots, \widehat{t}_{z_{p p}}\right)
$$


Esto es resultado de resolver el polinomio característico estimado a partir de la muestra $s$ de esta matriz:

$$
\widehat{S}=Z_{n}^{\prime} \Pi^{-1} Z_{n} \widehat{D}^{-1}=\widehat{B} \widehat{D}^{-1}
$$

Donde la matriz de Burt estimada

$$
\widehat{B}=Z_{n}^{\prime} \Pi^{-1} Z_{n}
$$

la matriz diagonal estimada obtenida a partir de la matriz de Burt, corresponde a una matriz de orden $(p, p)$, dada por:

$$
\widehat{D}=\operatorname{diag}\left\{Z_{n}^{\prime} \Pi^{-1} Z_{n}\right\}
$$

y $\Pi$ es la matriz diagonal de probabilidades de inclusión:

$$
\Pi=\operatorname{diag}\left\{\pi_{1}, \ldots, \pi_{n}\right\}
$$

Donde los factores de expansión que afectan la estimación son $f_{k}=\frac{1}{\pi_{k}}=\frac{N}{n}$

\subsection{Estimación mediante muestreo $\pi \mathrm{PT}$}

En este diseño se utiliza la información auxiliar disponible para todo $k \in U$ de una variable $X$ altamente correlacionada con la variable de estudio $Y$. Con ayuda de esta información, se construyen probabilidades proporcionales al tamaño $\pi_{k}$, para todos los elementos de la población y fijando de antemano el tamaño de muestra $n$, donde:

$$
\pi_{k}=\frac{n x_{k}}{\sum_{U} x_{k}}
$$

con factores de expansión:

$$
f_{k}=\frac{\sum_{U} x_{k}}{n x_{k}}
$$

para todo $k=1, \ldots, N$

\subsection{Estimación mediante muestreo bietápico $\pi$ PT-MAS}

En el caso en el que la primera etapa se realiza un diseño $\pi P T$ para seleccionar las UMP's tomando $n_{i}$ de los $N_{i}$ que componen la unidad, con:

$$
\pi_{I}=\frac{n_{I} x_{k}}{\sum_{U} x_{k}}
$$

Comunicaciones en Estadística, diciembre 2015, Vol. 8, No. 2 
Luego dentro de cada UMP se realiza un diseño MAS donde:

$$
\pi_{j}=\frac{n_{j}}{N_{j}}
$$

Se obtienen así los factores de expansión:

$$
f_{\text {exp }}=f_{1} * f_{2}=\frac{1}{\pi_{k}}=\frac{\left(\sum_{U} x_{k}\right)\left(N_{i \mid I}\right)}{\left(n_{I} x_{k}\right)\left(n_{i \mid I}\right)}
$$

para $k=1, \ldots, N$.

\section{Estudio de simulación}

Con el objetivo de realizar la estimación de los valores propios a través de los intervalos de confianza en el análisis de componentes principales a partir de una muestra probabilística, se tomó como la población objetivo la base de datos Finanzas.SBD del software SPAD, la cual contiene 468 registros de clientes de un Banco. La tiene registrada las variables: el tipo de cliente (Tipo) con dos modalidades, donde el $50.64 \%$ pertenece a la categoría 1 y el $49.36 \%$ está en la categoría 2; Edad tiene cuatro modalidades que están distribuidas en la población así: $18.80 \%$, $32.05 \%, 26.07 \%$ y $23.08 \%$ para las categorías 1, 2, 3 y 4, respectivamente; Estado Civil con las categorías 1, 2, 3 y 4, que cuentan con el $36.32 \%, 47.22 \% 13.03 \%$ y $3.42 \%$, respectivamente; Antigüedad en el trabajo, que cuenta con 5 categorías están presentes en la población con un $42.52 \%$ para la modalidad 1, $10.04 \%$ para la $2,14.74 \%$ para la $3,14.10 \%$ para la 4 y $18.59 \%$ en la categoría 5 : finalmente la variable Profesión que tiene tres modalidades con el $16.45 \% 50.64 \%$ y $32.91 \%$ en las modalidades 1,2 y 3 . Además, la base contiene la variable Cupo disponible, que se empleará como información auxiliar en los diseños de muestreo.

Para la realización del estudio de simulación mediante el software $\mathrm{R}$, se generó una población artificial de tamaño $N=20000$, mediante una distribución multinomial de tal forma que las proporciones descritas anteriormente se conservarán posteriormente se seleccionan muestras probabilísticas de tamaños $n=25,50$ y 100, mediante los diseños MAS y $\pi \mathrm{PT}$ en una etapa. A su vez, en dos etapas se utilizó el diseño $\pi$ PT-MAS, para emplear el diseño en dos etapas se definen 4 grupos aleatorios. La asignación de los elementos dentro de estos grupos se hizo de forma aleatoria, los tamaños de las muestras probabilística a seleccionar dentro de los grupos se hace mediante la asignación óptima. Se desea comparar la cobertura y la longitud promedio de los intervalos de confianzas construidos con la metodologías tradicionales y la propuesta; para ello, se calculan los intervalos de confianza para los valores propios $\lambda_{i}$ a partir de las muestras probabilísticas, luego se determina si el valor propio real (calculado a partir de la población) se encuentra dentro del intervalo de confianza y se mide su longitud. Este procedimiento se repite 1000 veces, con lo que se determina la cobertura y la longitud promedio de los intervalos 
de confianza construidos con estás metodologías. Para comparar los intervalos de confianza teniendo en cuenta de manera simultánea las cobertura y la amplitud se utiliza el índice de (Correa 2003), dado por:

$$
I=\frac{2-L P I}{2} \frac{N R}{N N}
$$

Donde LPI: longitud promedio de los intervalos de confianza, NR: nivel real de simulación, NN: nivel nominal, cuyos resultados se presentan en los anexos, utilizando un nivel nominal del $95 \%$.

\section{Resultados de simulación}

Se seleccionan tamaños de muestra $n=25$, 50 y 100 los resultados para los intervalos de confianza (I.C) asintóticos de Anderson, Bootstrap y Jackknife para los valores propios en un análisis de correspondencias a partir de una muestra probabilística. Estas son sus siglas; VP: valor propio, LONGPA: longitud del Intervalo de Anderson, LONGPB: longitud del Intervalo Bootstrap, LONGPJ: longitud del Intervalo Jackknife, TCA: cobertura I.C Anderson, TCB: cobertura I.C Bootstrap, TCJ: cobertura I.C Jackknife, utilizando un nivel de confianza nominal del $95 \%$, se presentan a continuación:

\subsection{Resultados diseño MAS}

Tabla 1: Resultados para $n=25$

\begin{tabular}{|c|c|c|c|c|c|c|c|}
\hline Eje & VP & LONGPA & TCA & LONGPB & TCB & LONGPJ & TCJ \\
\hline 1 & 0,410 & 0,825 & $\mathbf{0 , 9 8 6}$ & $\mathbf{0 , 1 5 4}$ & 0,393 & 0,262 & 0,716 \\
2 & 0,319 & 0,661 & $\mathbf{0 , 9 8 9}$ & $\mathbf{0 , 1 0 3}$ & 0,141 & 0,236 & 0,796 \\
3 & 0,272 & 0,550 & $\mathbf{0 , 9 9 8}$ & $\mathbf{0 , 0 7 9}$ & 0,151 & 0,207 & 0,859 \\
4 & 0,258 & 0,463 & $\mathbf{1 , 0 0 0}$ & $\mathbf{0 , 0 6 0}$ & 0,576 & 0,184 & 0,994 \\
5 & 0,253 & 0,391 & $\mathbf{1 , 0 0 0}$ & $\mathbf{0 , 0 4 8}$ & 0,758 & 0,182 & 0,992 \\
6 & 0,248 & 0,317 & $\mathbf{0 , 9 9 7}$ & $\mathbf{0 , 0 6 0}$ & 0,114 & 0,201 & 0,855 \\
7 & 0,214 & 0,244 & $\mathbf{0 , 9 7 9}$ & $\mathbf{0 , 0 5 8}$ & 0,029 & 0,185 & 0,700 \\
8 & 0,197 & 0,179 & $\mathbf{0 , 8 0 6}$ & $\mathbf{0 , 0 4 8}$ & 0,000 & 0,173 & 0,475 \\
9 & 0,175 & 0,120 & $\mathbf{0 , 4 5 4}$ & $\mathbf{0 , 0 3 6}$ & 0,000 & 0,140 & 0,261 \\
10 & 0,153 & 0,085 & $\mathbf{0 , 1 7 5}$ & $\mathbf{0 , 0 2 3}$ & 0,000 & 0,099 & 0,084 \\
\hline
\end{tabular}

Es importante resaltar que los valores en negrilla corresponden a los mejores resultados, para cada valor propio.

Se nota que mediante el diseño de muestreo MAS, las tasas de cobertura de los intervalos de confianza de Anderson resultan ser mayores; sin embargo, resultan ser más amplios, por tener menor amplitud los intervalos de confianza Boostrap. Por otra parte, teniendo en cuenta los dos aspectos simultáneamente: tasas de co- 
Tabla 2: Resultados para $n=50$

\begin{tabular}{|c|c|c|c|c|c|c|c|}
\hline Eje & VP & LONGPA & TCA & LONGPB & TCB & LONGPJ & TCJ \\
\hline 1 & 0,410 & 0,431 & $\mathbf{0 , 9 8 9}$ & $\mathbf{0 , 1 2 2}$ & 0,754 & 0,190 & 0,854 \\
2 & 0,319 & 0,346 & $\mathbf{0 , 9 9 6}$ & $\mathbf{0 , 0 7 9}$ & 0,514 & 0,161 & 0,883 \\
3 & 0,272 & 0,301 & $\mathbf{0 , 9 9 6}$ & $\mathbf{0 , 0 6 1}$ & 0,306 & 0,150 & 0,861 \\
4 & 0,258 & 0,264 & $\mathbf{1 , 0 0 0}$ & $\mathbf{0 , 0 4 7}$ & 0,662 & 0,134 & 0,993 \\
5 & 0,253 & 0,233 & $\mathbf{1 , 0 0 0}$ & $\mathbf{0 , 0 3 7}$ & 0,743 & 0,119 & 0,999 \\
6 & 0,248 & 0,204 & $\mathbf{1 , 0 0 0}$ & $\mathbf{0 , 0 4 3}$ & 0,103 & 0,131 & 0,944 \\
7 & 0,214 & 0,173 & $\mathbf{0 , 9 9 6}$ & $\mathbf{0 , 0 4 5}$ & 0,142 & 0,133 & 0,906 \\
8 & 0,197 & 0,143 & $\mathbf{0 , 9 7 1}$ & $\mathbf{0 , 0 4 2}$ & 0,029 & 0,130 & 0,749 \\
9 & 0,175 & 0,113 & $\mathbf{0 , 8 1 4}$ & $\mathbf{0 , 0 3 8}$ & 0,002 & 0,125 & 0,592 \\
10 & 0,153 & 0,085 & $\mathbf{0 , 4 8 1}$ & $\mathbf{0 , 0 3 2}$ & 0,000 & 0,098 & 0,329 \\
\hline
\end{tabular}

Tabla 3: Resultados para $n=100$

\begin{tabular}{|c|c|c|c|c|c|c|c|}
\hline Eje & VP & LONGPA & TCA & LONGPB & TCB & LONGPJ & TCJ \\
\hline 1 & 0,410 & 0,262 & $\mathbf{0 , 9 9 5}$ & $\mathbf{0 , 0 9 1}$ & 0,883 & 0,137 & 0,934 \\
2 & 0,319 & 0,208 & $\mathbf{1 , 0 0 0}$ & $\mathbf{0 , 0 5 9}$ & 0,794 & 0,115 & 0,952 \\
3 & 0,272 & 0,183 & $\mathbf{0 , 9 9 5}$ & $\mathbf{0 , 0 4 5}$ & 0,549 & 0,104 & 0,928 \\
4 & 0,258 & 0,166 & $\mathbf{1 , 0 0 0}$ & $\mathbf{0 , 0 3 5}$ & 0,803 & 0,093 & 0,991 \\
5 & 0,253 & 0,151 & $\mathbf{1 , 0 0 0}$ & $\mathbf{0 , 0 2 8}$ & 0,733 & 0,082 & 1,000 \\
6 & 0,248 & 0,138 & $\mathbf{1 , 0 0 0}$ & $\mathbf{0 , 0 3 2}$ & 0,097 & 0,092 & 0,959 \\
7 & 0,214 & 0,122 & $\mathbf{1 , 0 0 0}$ & $\mathbf{0 , 0 3 4}$ & 0,359 & 0,096 & 0,976 \\
8 & 0,197 & 0,106 & $\mathbf{0 , 9 9 7}$ & $\mathbf{0 , 0 3 3}$ & 0,133 & 0,094 & 0,931 \\
9 & 0,175 & 0,090 & $\mathbf{0 , 9 6 1}$ & $\mathbf{0 , 0 3 1}$ & 0,056 & 0,088 & 0,847 \\
10 & 0,153 & 0,074 & $\mathbf{0 , 8 0 9}$ & $\mathbf{0 , 0 3 0}$ & 0,026 & 0,078 & 0,684 \\
\hline
\end{tabular}

bertura y amplitud de los intervalos de confianza, el método Jacknnife resulta ser más eficiente. Esto, se cumple para los cinco primeros ejes factoriales (ver anexo). Es importante señalar que las tendencias de mayor cobertura y menor longitud promedio de los intervalos de confianza se presentan en todos los tamaños muestrales $n=25,50, y 100$.

Ahora, realizando la estimación mediante un diseño de muestreo probabilístico $\pi \mathrm{PT}$, se obtienen los resultados que se muestran a continuación:

\subsection{Resultados diseño $\pi \mathrm{PT}$}

De igual forma, se nota que las tasas de cobertura de los intervalos de confianza de Anderson resultan ser mayores; además, las longitudes promedios de los intervalos mediante remuestreo Boostrap son menores, lo que se cumple para todos los ejes factoriales y tamaños de muestra en este estudio. 
Tabla 4: Resultados para $n=25$

\begin{tabular}{|c|c|c|c|c|c|c|c|}
\hline Eje & VP & LONGPA & TCA & LONGPB & TCB & LONGPJ & TCJ \\
\hline 1 & 0,410 & 0,841 & $\mathbf{0 , 9 7 0}$ & $\mathbf{0 , 1 6 4}$ & 0,375 & 0,278 & 0,713 \\
2 & 0,319 & 0,663 & $\mathbf{0 , 9 8 5}$ & $\mathbf{0 , 1 0 8}$ & 0,138 & 0,237 & 0,758 \\
3 & 0,272 & 0,549 & $\mathbf{0 , 9 8 9}$ & $\mathbf{0 , 0 8 1}$ & 0,138 & 0,214 & 0,885 \\
4 & 0,258 & 0,460 & $\mathbf{1 , 0 0 0}$ & $\mathbf{0 , 0 6 2}$ & 0,531 & 0,183 & 0,996 \\
5 & 0,253 & 0,383 & $\mathbf{1 , 0 0 0}$ & $\mathbf{0 , 0 5 1}$ & 0,711 & 0,191 & 0,981 \\
6 & 0,248 & 0,306 & $\mathbf{0 , 9 9 9}$ & $\mathbf{0 , 0 6 3}$ & 0,098 & 0,197 & 0,803 \\
7 & 0,214 & 0,235 & $\mathbf{0 , 9 5 9}$ & $\mathbf{0 , 0 5 9}$ & 0,042 & 0,185 & 0,657 \\
8 & 0,197 & 0,169 & $\mathbf{0 , 7 3 6}$ & $\mathbf{0 , 0 4 8}$ & 0,000 & 0,171 & 0,422 \\
9 & 0,175 & 0,112 & $\mathbf{0 , 3 6 6}$ & $\mathbf{0 , 0 3 5}$ & 0,000 & 0,135 & 0,209 \\
10 & 0,153 & 0,081 & $\mathbf{0 , 1 5 2}$ & $\mathbf{0 , 0 2 2}$ & 0,000 & 0,101 & 0,079 \\
\hline
\end{tabular}

Tabla 5: Resultados para $n=50$

\begin{tabular}{|c|c|c|c|c|c|c|c|}
\hline Eje & VP & LONGPA & TCA & LONGPB & TCB & LONGPJ & TCJ \\
\hline 1 & 0,410 & 0,438 & $\mathbf{0 , 9 8 2}$ & $\mathbf{0 , 1 2 8}$ & 0,738 & 0,200 & 0,852 \\
2 & 0,319 & 0,350 & $\mathbf{0 , 9 9 3}$ & $\mathbf{0 , 0 8 3}$ & 0,464 & 0,167 & 0,843 \\
3 & 0,272 & 0,303 & $\mathbf{0 , 9 9 0}$ & $\mathbf{0 , 0 6 3}$ & 0,279 & 0,155 & 0,857 \\
4 & 0,258 & 0,265 & $\mathbf{1 , 0 0 0}$ & $\mathbf{0 , 0 4 8}$ & 0,642 & 0,137 & 0,994 \\
5 & 0,253 & 0,232 & $\mathbf{1 , 0 0 0}$ & $\mathbf{0 , 0 3 8}$ & 0,746 & 0,122 & 0,998 \\
6 & 0,248 & 0,201 & $\mathbf{1 , 0 0 0}$ & $\mathbf{0 , 0 4 5}$ & 0,106 & 0,137 & 0,932 \\
7 & 0,214 & 0,169 & $\mathbf{0 , 9 9 5}$ & $\mathbf{0 , 0 4 7}$ & 0,117 & 0,139 & 0,901 \\
8 & 0,197 & 0,138 & $\mathbf{0 , 9 3 0}$ & $\mathbf{0 , 0 4 3}$ & 0,010 & 0,131 & 0,684 \\
9 & 0,175 & 0,109 & $\mathbf{0 , 7 1 8}$ & $\mathbf{0 , 0 3 7}$ & 0,002 & 0,124 & 0,521 \\
10 & 0,153 & 0,083 & $\mathbf{0 , 4 3 5}$ & $\mathbf{0 , 0 3 2}$ & 0,000 & 0,098 & 0,280 \\
\hline
\end{tabular}

Tabla 6: Resultados para $n=100$

\begin{tabular}{|c|c|c|c|c|c|c|c|}
\hline Eje & VP & LONGPA & TCA & LONGPB & TCB & LONGPJ & TCJ \\
\hline 1 & 0,410 & 0,264 & $\mathbf{0 , 9 9 3}$ & $\mathbf{0 , 0 9 6}$ & 0,876 & 0,144 & 0,930 \\
2 & 0,319 & 0,210 & $\mathbf{0 , 9 9 7}$ & $\mathbf{0 , 0 6 2}$ & 0,778 & 0,120 & 0,937 \\
3 & 0,272 & 0,185 & $\mathbf{0 , 9 9 1}$ & $\mathbf{0 , 0 4 7}$ & 0,493 & 0,110 & 0,901 \\
4 & 0,258 & 0,166 & $\mathbf{1 , 0 0 0}$ & $\mathbf{0 , 0 3 6}$ & 0,785 & 0,097 & 0,993 \\
5 & 0,253 & 0,151 & $\mathbf{1 , 0 0 0}$ & $\mathbf{0 , 0 2 8}$ & 0,724 & 0,085 & 1,000 \\
6 & 0,248 & 0,137 & $\mathbf{1 , 0 0 0}$ & $\mathbf{0 , 0 3 3}$ & 0,115 & 0,097 & 0,972 \\
7 & 0,214 & 0,121 & $\mathbf{0 , 9 9 8}$ & $\mathbf{0 , 0 3 5}$ & 0,298 & 0,101 & 0,979 \\
8 & 0,197 & 0,104 & $\mathbf{0 , 9 8 9}$ & $\mathbf{0 , 0 3 4}$ & 0,084 & 0,097 & 0,897 \\
9 & 0,175 & 0,088 & $\mathbf{0 , 9 3 6}$ & $\mathbf{0 , 0 3 2}$ & 0,037 & 0,093 & 0,818 \\
10 & 0,153 & 0,072 & $\mathbf{0 , 7 5 8}$ & $\mathbf{0 , 0 3 0}$ & 0,011 & 0,079 & 0,638 \\
\hline
\end{tabular}

\subsection{Resultados diseño $\pi$ PT-MAS}

Finalmente, mediante el diseño de muestreo probabilístico bietápico $\pi$ PT-MAS, se sigue confirmando las tendencias reflejadas en los demás diseños de muestreo 
236Javier Ramírez Montoya, Guillermo Martínez Flórez \& Stalyn Guerrero Gómez

utilizados.

Tabla 7: Resultados para $n=25$

\begin{tabular}{|c|c|c|c|c|c|c|c|}
\hline Eje & VP & LONGPA & TCA & LONGPB & TCB & LONGPJ & TCJ \\
\hline 1 & 0,410 & 0,826 & $\mathbf{0 , 9 6 6}$ & $\mathbf{0 , 1 5 8}$ & 0,366 & 0,265 & 0,739 \\
2 & 0,319 & 0,652 & $\mathbf{0 , 9 9 1}$ & $\mathbf{0 , 1 0 6}$ & 0,173 & 0,229 & 0,759 \\
3 & 0,272 & 0,544 & $\mathbf{0 , 9 9 7}$ & $\mathbf{0 , 0 8 1}$ & 0,153 & 0,207 & 0,892 \\
4 & 0,258 & 0,457 & $\mathbf{1 , 0 0 0}$ & $\mathbf{0 , 0 6 0}$ & 0,574 & 0,178 & 0,996 \\
5 & 0,253 & 0,383 & $\mathbf{1 , 0 0 0}$ & $\mathbf{0 , 0 4 7}$ & 0,702 & 0,181 & 0,972 \\
6 & 0,248 & 0,310 & $\mathbf{0 , 9 9 4}$ & $\mathbf{0 , 0 6 1}$ & 0,090 & 0,202 & 0,818 \\
7 & 0,214 & 0,237 & $\mathbf{0 , 9 5 9}$ & $\mathbf{0 , 0 5 9}$ & 0,053 & 0,189 & 0,690 \\
8 & 0,197 & 0,172 & $\mathbf{0 , 7 8 2}$ & $\mathbf{0 , 0 4 8}$ & 0,000 & 0,174 & 0,439 \\
9 & 0,175 & 0,116 & $\mathbf{0 , 3 9 6}$ & $\mathbf{0 , 0 3 6}$ & 0,000 & 0,136 & 0,208 \\
10 & 0,153 & 0,081 & $\mathbf{0 , 1 4 0}$ & $\mathbf{0 , 0 2 3}$ & 0,000 & 0,100 & 0,088 \\
\hline
\end{tabular}

Tabla 8: Resultados para $n=50$

\begin{tabular}{|c|c|c|c|c|c|c|c|}
\hline Eje & VP & LONGPA & TCA & LONGPB & TCB & LONGPJ & TCJ \\
\hline 1 & 0,410 & 0,429 & $\mathbf{0 , 9 7 2}$ & $\mathbf{0 , 1 2 3}$ & 0,738 & 0,188 & 0,845 \\
2 & 0,319 & 0,345 & $\mathbf{0 , 9 9 4}$ & $\mathbf{0 , 0 8 1}$ & 0,521 & 0,161 & 0,857 \\
3 & 0,272 & 0,298 & $\mathbf{0 , 9 9 3}$ & $\mathbf{0 , 0 6 2}$ & 0,341 & 0,148 & 0,881 \\
4 & 0,258 & 0,262 & $\mathbf{1 , 0 0 0}$ & $\mathbf{0 , 0 4 6}$ & 0,691 & 0,128 & 0,994 \\
5 & 0,253 & 0,232 & $\mathbf{1 , 0 0 0}$ & $\mathbf{0 , 0 3 6}$ & 0,738 & 0,118 & 0,999 \\
6 & 0,248 & 0,202 & $\mathbf{1 , 0 0 0}$ & $\mathbf{0 , 0 4 3}$ & 0,108 & 0,133 & 0,919 \\
7 & 0,214 & 0,171 & $\mathbf{0 , 9 9 3}$ & $\mathbf{0 , 0 4 6}$ & 0,160 & 0,135 & 0,891 \\
8 & 0,197 & 0,140 & $\mathbf{0 , 9 5 0}$ & $\mathbf{0 , 0 4 3}$ & 0,030 & 0,131 & 0,712 \\
9 & 0,175 & 0,110 & $\mathbf{0 , 7 5 0}$ & $\mathbf{0 , 0 3 7}$ & 0,003 & 0,120 & 0,528 \\
10 & 0,153 & 0,086 & $\mathbf{0 , 5 1 9}$ & $\mathbf{0 , 0 3 2}$ & 0,000 & 0,100 & 0,354 \\
\hline
\end{tabular}

Tabla 9: Resultados para $n=100$

\begin{tabular}{|c|c|c|c|c|c|c|c|}
\hline Eje & VP & LONGPA & TCA & LONGPB & TCB & LONGPJ & TCJ \\
\hline 1 & 0,410 & 0,258 & $\mathbf{0 , 9 7 1}$ & $\mathbf{0 , 0 8 9}$ & 0,721 & 0,133 & 0,861 \\
2 & 0,319 & 0,207 & $\mathbf{0 , 9 9 8}$ & $\mathbf{0 , 0 5 9}$ & 0,764 & 0,115 & 0,949 \\
3 & 0,272 & 0,182 & $\mathbf{0 , 9 9 9}$ & $\mathbf{0 , 0 4 5}$ & 0,552 & 0,105 & 0,945 \\
4 & 0,258 & 0,165 & $\mathbf{1 , 0 0 0}$ & $\mathbf{0 , 0 3 4}$ & 0,800 & 0,092 & 0,993 \\
5 & 0,253 & 0,151 & $\mathbf{1 , 0 0 0}$ & $\mathbf{0 , 0 2 7}$ & 0,749 & 0,082 & 1,000 \\
6 & 0,248 & 0,137 & $\mathbf{1 , 0 0 0}$ & $\mathbf{0 , 0 3 1}$ & 0,120 & 0,091 & 0,928 \\
7 & 0,214 & 0,122 & $\mathbf{1 , 0 0 0}$ & $\mathbf{0 , 0 3 4}$ & 0,438 & 0,101 & 0,952 \\
8 & 0,197 & 0,105 & $\mathbf{0 , 9 8 1}$ & $\mathbf{0 , 0 3 4}$ & 0,172 & 0,097 & 0,858 \\
9 & 0,175 & 0,089 & $\mathbf{0 , 9 2 7}$ & $\mathbf{0 , 0 3 2}$ & 0,077 & 0,093 & 0,790 \\
10 & 0,153 & 0,075 & $\mathbf{0 , 8 2 7}$ & $\mathbf{0 , 0 3 0}$ & 0,042 & 0,079 & 0,716 \\
\hline
\end{tabular}




\section{Conclusiones}

- Cuando se realiza el procedimiento de estimación de los valores propios mediante muestreo probabilístico en el análisis de correspondencias, es recomendable realizar la complementación mediante el calculo de los intervalos de confianza, teniendo en cuenta la estrategia más eficiente en este estudio, logrando una mejor decisión en la escogencia de planos factoriales.

- Los intervalos de confianza para los valores propios en el análisis de correspondencias a partir de una muestra probabilística mediante el método de remuestreo Bootstrap resultan tener menor longitud, pero sesgados en sus estimaciones, reflejados en su cobertura.

- Los resultados de los intervalos de confianza mediante remuestreo Jackknife para los diseños de muestreo probabilístico MAS, $\pi \mathrm{PT}$ y $\pi \mathrm{PT}-\mathrm{MAS}$, tienen mejores resultados teniendo en cuenta la longitud y las tasas de coberturas Se resalta que en el diseño de muestreo $\pi$ PT-MAS se debe tener cuidado en la escogencia de la variable auxiliar para el calculo de los factores de expansión.

- El índice de Correa et al. (2003) puede ser utilizado para comparar los intervalos de confianza de los valores propios en el análisis de correspondencias a partir de una muestra probabilística.

Recibido: 20 de agosto del 2015 Aceptado: 13 de octubre del 2015

\section{Referencias}

Correa, J. \& Sierra, E. (2003), 'Intervalos de confianza para la comparación de dos proporciones', Revista Colombiana de Estadistica 26, 61-75.

Milan, L. \& Whittaker, R. (1995), 'Application of the parametric bootstrap to models that incorporate a singular value decomposition', Applied Statistics 44, 31-49.

R Core Team (2014), R: A Language and Environment for Statistical Computing, $\mathrm{R}$ Foundation for Statistical Computing, Vienna, Austria.

*http://www.R-project.org/

Ramirez, J. \& Martinez, G. (2010), 'Análisis de Correspondencias a partir de una muestra probabilística', Revista Colombiana de Estadística 33, 273-293.

Sarndal, C., S. B. . W. J. (2003), Model Assisted Survey Sampling, Springer-Verlag, New York.

Wolter, K. (1985), Introduction to Variance Estimation, Springer-Verlag, Berlin. 
238Javier Ramírez Montoya, Guillermo Martínez Flórez \& Stalyn Guerrero Gómez

\section{A. Tablas}

Tabla 10: Resultados Indices para $M A S$

\begin{tabular}{|c|c|c|c|c|c|c|c|c|}
\hline \multicolumn{3}{c}{$\mathrm{n}=25$} & \multicolumn{3}{c}{$\mathrm{n}=50$} & \multicolumn{3}{c}{$\mathrm{n}=100$} \\
\hline And. & Boot. & Jack. & And. & Boot. & Jack. & And. & Boot. & Jack. \\
\hline 0,610 & 0,382 & 0,655 & 0,817 & 0,745 & 0,814 & 0,963 & 0,920 & 0,988 \\
0,697 & 0,141 & 0,739 & 0,867 & 0,520 & 0,855 & 0,981 & 0,889 & 0,991 \\
0,762 & 0,153 & 0,811 & 0,891 & 0,312 & 0,838 & 0,991 & 0,856 & 0,990 \\
0,809 & 0,588 & 0,950 & 0,914 & 0,680 & 0,975 & 0,995 & 0,927 & 1,017 \\
0,847 & 0,779 & 0,949 & 0,930 & 0,768 & 0,989 & 0,998 & 0,769 & 1,024 \\
0,883 & 0,116 & 0,810 & 0,945 & 0,106 & 0,929 & 1,002 & 0,159 & 0,997 \\
0,905 & 0,030 & 0,669 & 0,958 & 0,146 & 0,890 & 1,007 & 0,603 & 1,012 \\
0,772 & 0,000 & 0,457 & 0,949 & 0,030 & 0,737 & 1,012 & 0,347 & 1,001 \\
0,449 & 0,000 & 0,256 & 0,808 & 0,002 & 0,584 & 1,013 & 0,300 & 0,995 \\
0,176 & 0,000 & 0,084 & 0,485 & 0,000 & 0,329 & 0,989 & 0,182 & 0,951 \\
\hline
\end{tabular}

Tabla 11: Resultados Indices para $\pi P T$

\begin{tabular}{|c|c|c|c|c|c|c|c|c|}
\hline \multicolumn{3}{c}{$\mathrm{n}=25$} & $\mathrm{n}=50$ & \multicolumn{3}{c}{$\mathrm{n}=100$} \\
\hline And. & Boot. & Jack. & And. & Boot. & Jack. & And. & Boot. & Jack. \\
\hline 0,592 & 0,362 & 0,646 & 0,807 & 0,727 & 0,807 & 0,907 & 0,878 & 0,908 \\
0,693 & 0,137 & 0,703 & 0,862 & 0,468 & 0,813 & 0,939 & 0,794 & 0,927 \\
0,755 & 0,139 & 0,832 & 0,884 & 0,284 & 0,832 & 0,947 & 0,507 & 0,896 \\
0,811 & 0,542 & 0,952 & 0,913 & 0,660 & 0,975 & 0,965 & 0,811 & 0,995 \\
0,851 & 0,729 & 0,934 & 0,931 & 0,770 & 0,986 & 0,973 & 0,751 & 1,008 \\
0,891 & 0,100 & 0,762 & 0,947 & 0,109 & 0,914 & 0,981 & 0,119 & 0,974 \\
0,891 & 0,043 & 0,628 & 0,959 & 0,120 & 0,883 & 0,987 & 0,308 & 0,978 \\
0,709 & 0,000 & 0,406 & 0,911 & 0,010 & 0,673 & 0,987 & 0,087 & 0,898 \\
0,364 & 0,000 & 0,205 & 0,715 & 0,002 & 0,514 & 0,942 & 0,038 & 0,821 \\
0,154 & 0,000 & 0,079 & 0,439 & 0,000 & 0,280 & 0,769 & 0,011 & 0,645 \\
\hline
\end{tabular}

\section{B. Códigos}

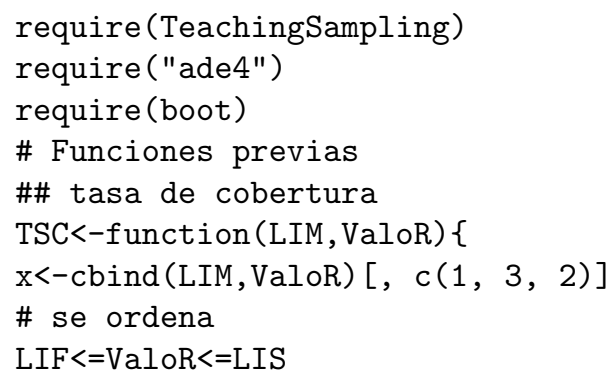


Tabla 12: Resultados Indices para $\pi P T-M A S$

\begin{tabular}{|c|c|c|c|c|c|c|c|c|}
\hline \multicolumn{3}{c}{$\mathrm{n}=25$} & \multicolumn{1}{c}{$\mathrm{n}=50$} & \multicolumn{3}{c}{$\mathrm{n}=100$} \\
\hline And. & Boot. & Jack. & And. & Boot. & Jack. & And. & Boot. & Jack. \\
\hline 0,597 & 0,355 & 0,675 & 0,804 & 0,729 & 0,806 & 0,890 & 0,725 & 0,846 \\
0,703 & 0,172 & 0,707 & 0,866 & 0,526 & 0,829 & 0,942 & 0,780 & 0,942 \\
0,764 & 0,155 & 0,842 & 0,890 & 0,348 & 0,859 & 0,956 & 0,568 & 0,943 \\
0,812 & 0,586 & 0,955 & 0,915 & 0,711 & 0,979 & 0,966 & 0,828 & 0,997 \\
0,851 & 0,722 & 0,931 & 0,931 & 0,763 & 0,990 & 0,973 & 0,778 & 1,009 \\
0,884 & 0,092 & 0,774 & 0,946 & 0,111 & 0,903 & 0,981 & 0,124 & 0,932 \\
0,890 & 0,054 & 0,658 & 0,956 & 0,165 & 0,875 & 0,988 & 0,453 & 0,951 \\
0,752 & 0,000 & 0,422 & 0,930 & 0,031 & 0,700 & 0,978 & 0,178 & 0,859 \\
0,393 & 0,000 & 0,204 & 0,746 & 0,003 & 0,522 & 0,932 & 0,080 & 0,793 \\
0,141 & 0,000 & 0,088 & 0,523 & 0,000 & 0,354 & 0,838 & 0,044 & 0,724 \\
\hline
\end{tabular}

(apply ( $x, M A R G=1$, function ( $\mathrm{x}$ )

ifelse $(x[1]<=x[2] \& x[2]<=x[3], 1,0)))$

\}

\#\# Estimación Boot

hat.V.p $<-$ function $(z . s, i)\{$

$z . s b<-z . s[i$,

$(p<-$ dudi.coa $(z . s b$, scann $=$ FALSE $)$ \$eig $[1: 10])$

\}

\#\# Estimación jackknife

jackknife<-function (z.s, conf $=0.95)$

\{

thetahat <- dudi.coa(z.s, scann = FALSE) \$eig $[1: 10]$

$\mathrm{n}<-$ length (thetahat)

$\mathrm{u}<-\operatorname{matrix}(\mathrm{NA}, \mathrm{ncol}=\mathrm{n}, \mathrm{nrow}=\mathrm{nrow}(\mathrm{z} . \mathrm{s}))$

for (i in 1 :nrow (z.s)) \{

$u[i]<$,- dudi.coa(z.s $[-i]$, scann = FALSE) \$eig $[1: 10]$

\}

$\# \mathrm{u}<-$ na. omit $(\mathrm{u})$

$\mathrm{mJ}<-\operatorname{colMeans}(\mathrm{u}, \mathrm{na} \cdot \mathrm{rm}=\mathrm{T})$

$\operatorname{vjk}<-\operatorname{sqrt}(((\mathrm{n}-1) /(\mathrm{n})) * \operatorname{rowSums}((\mathrm{t}(\mathrm{u})-\mathrm{mJ}) \wedge 2, \mathrm{na} \cdot \mathrm{rm}=\mathrm{T}))$

ICJ<-cbind (LIF=thetahat-qnorm ( (1-conf) $/ 2$, lower. tail=F) $* v j k$,

LIS=thetahat+qnorm $((1-\operatorname{conf}) / 2$, lower .tail $=F) * v j k$,

Valor.P $=\mathrm{mJ}$ )

return (ICJ)

\}

Sim.MAS <- function (datos, $\mathrm{n}$, conf $=0.95)\{$

$\mathrm{N}<-$ nrow (datos)

$\mathrm{x}<-$ datos $[,-\mathrm{c}(2,6)]$

\# obtencion de la matrix $z$ 
240Javier Ramírez Montoya, Guillermo Martínez Flórez \& Stalyn Guerrero Gómez

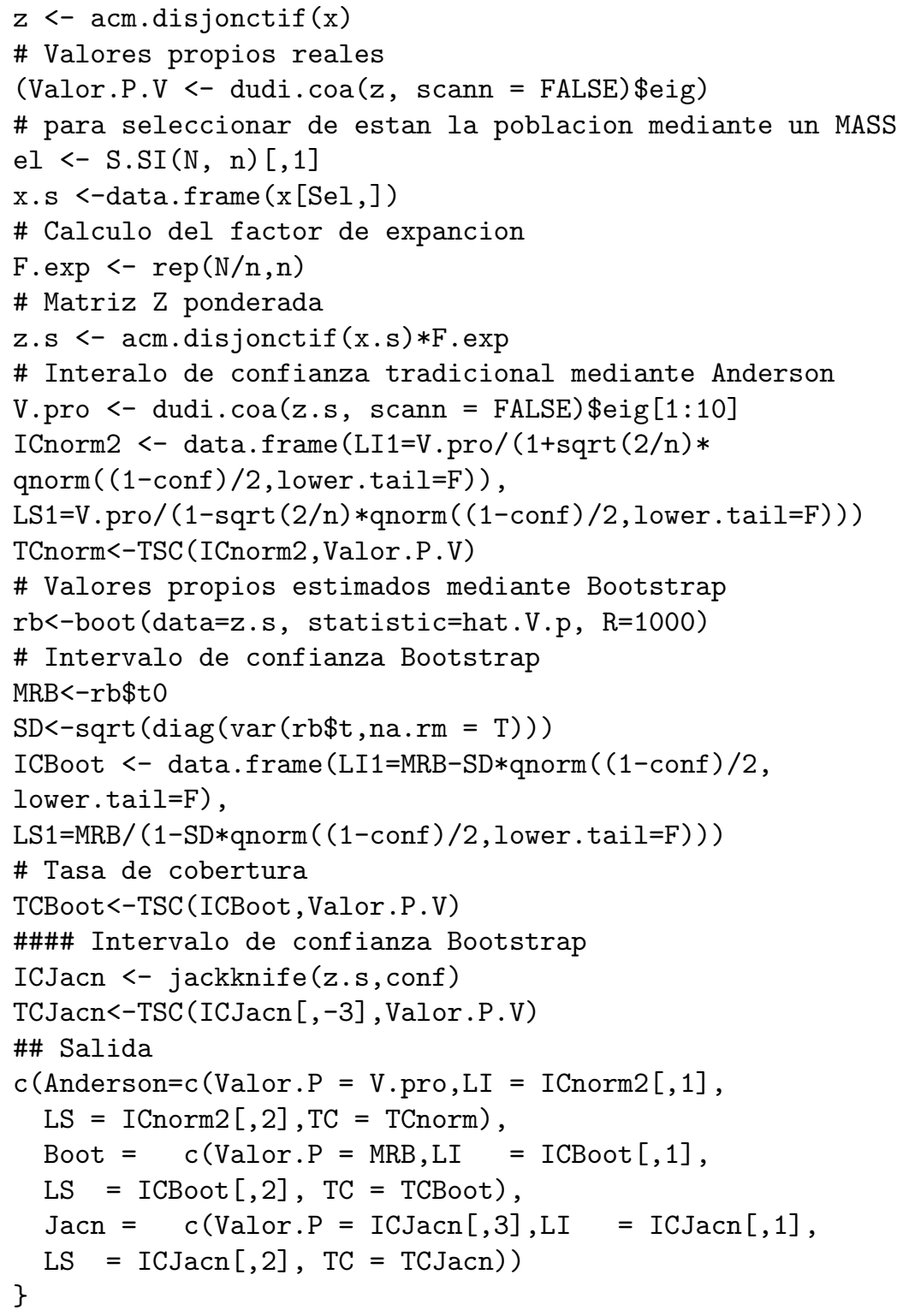




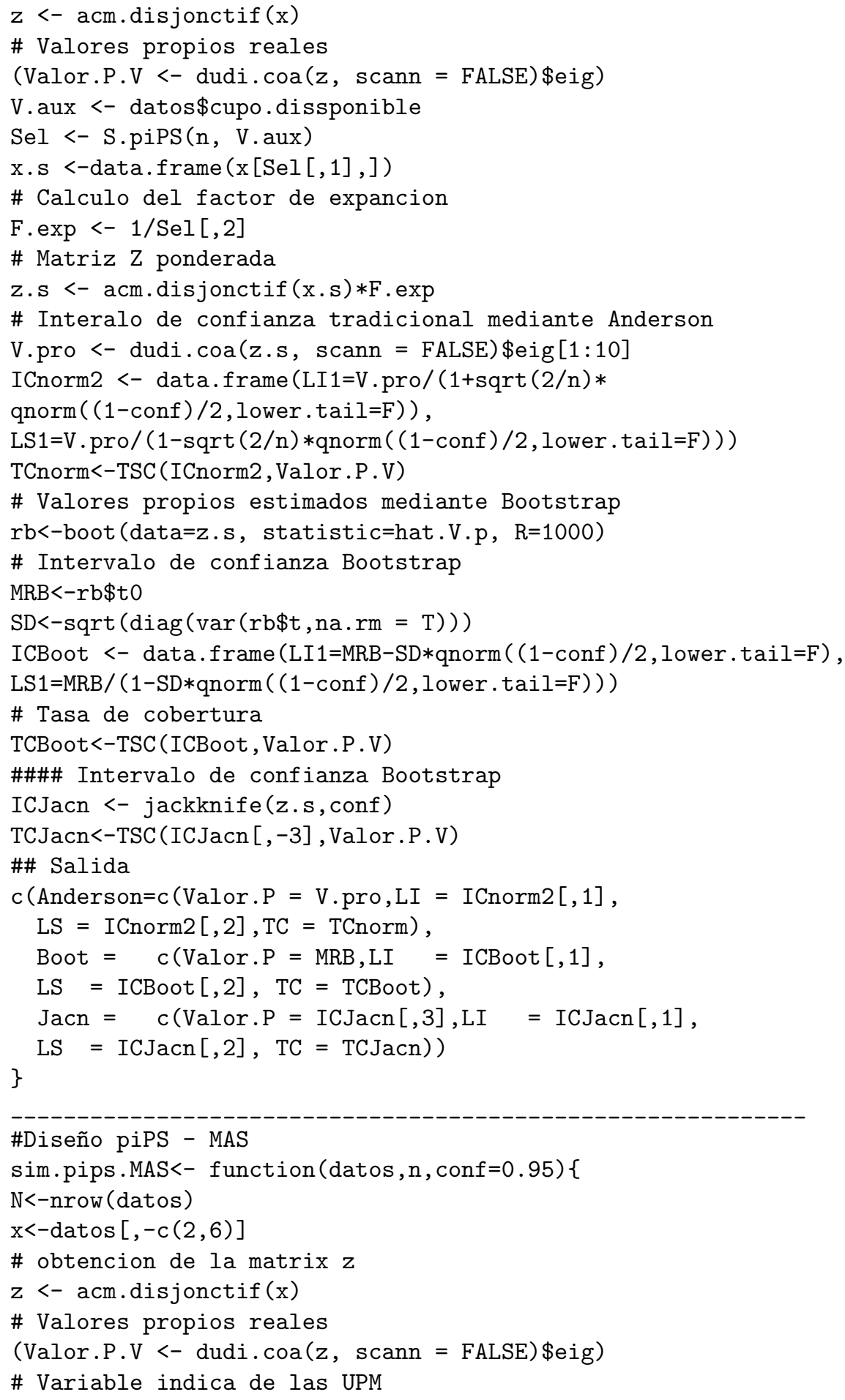


242Javier Ramírez Montoya, Guillermo Martínez Flórez \& Stalyn Guerrero Gómez

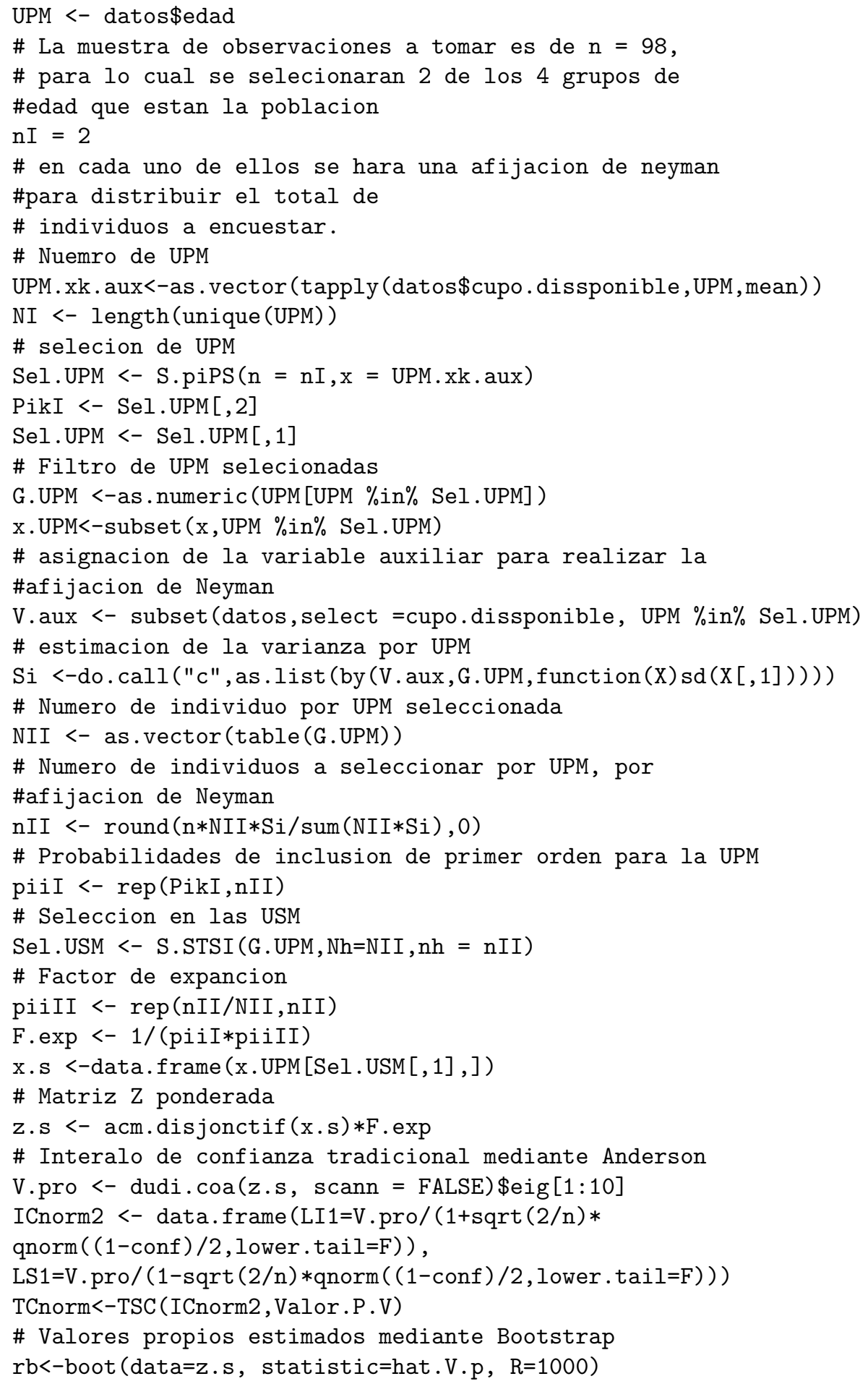




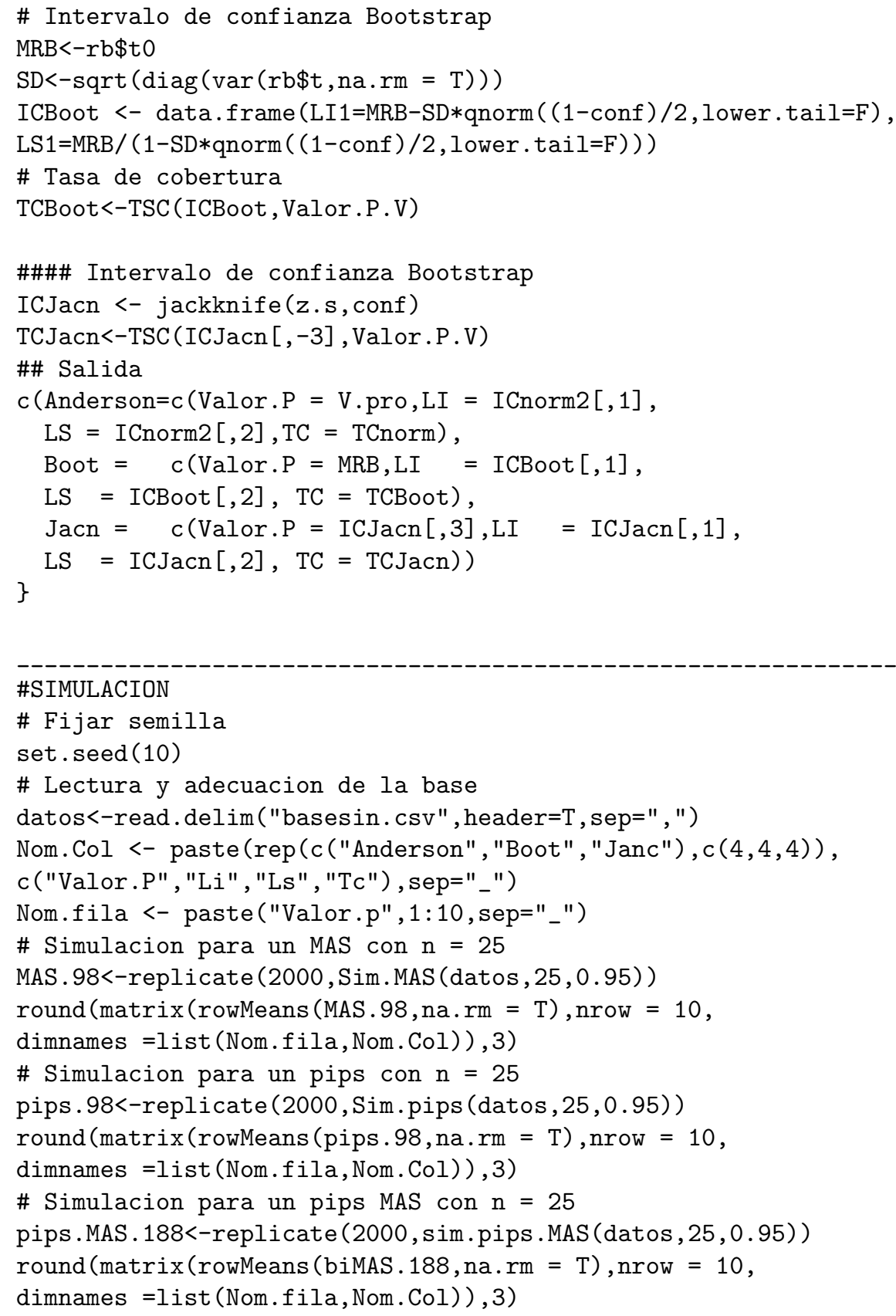

